\title{
Trema micrantha Blume na alimentação animal. \\ II - Degradaçào "in vitro" dos componentes da parede celular da periquiteira (Trema micrantha Blume), capim Aripuanà (Leptochloa domingensis Trin.) e capim elefante (Pennisetum purpureum Schum) $\left(^{*}\right)$
}

\author{
Pedro de Andrade ("*) \\ Paulo de Figueiredo Vieira $(* *$ ) \\ Luiz Claudio de A. Rosa (**) \\ Antonio Tadeu de Andrade $(* * * *)$
}

\section{Resumo}

Este trabalho estuda o potencial de certas espécies vegetais: piriquiteira (Trema micrantha Blume) e capim Aripuanã (Leptochloa domingensis Trin), que crescem espontaneamente no Núcleo de Pesquisa de Aripuanã, MT, na nutrição animal. Determinou-se a degradação "in vitro" da fibra dessas espécies. Foram analisadas as seguintes frações: fibra em detergente neutro (FDN), fibra em detergente ácido (FDA), celulose (Cel.) e lignina (Lig.). Uma amostra de capim e!efante com 35 dias de vegetação foi utilizada como referência. As quantidades de fibra em detergente neutro da Trema apresentaram-se constantes ao longo dos tempos de fermentação ao passo que para as gramíneas capim Aripuanã e capim elefante, a referida fração (FDN) apresentou digestibilidade variando de $40,36 \%$ a $46,84 \%$ às 48 horas de fermentação. Com relação à fibra em detergente ácido, observou-se quase o mesmo comportamento da fibra em detergente neutro, pois a digestibilidade dessa fração (FDA), para as gramíneas variou de 40 a $65 \%$, respectivamente, para capim Aripuanã e elefante enquanto que para a Trema não houve diferença significativa. A provável explicação para esses fatos é que a Trema apresenta teores de lignina três vezes maiores que os teores de lignina das gramíneas, dificultando a atuação dos microorganismos. Conclui-se que a Trema pode ser útil na alimentação de bovinos desde que se utilizem partes da planta que apresentem baixos teores de fibra. O capim Aripuanã apresenta um comportamento semelhante ao do capim elefante.
INTRODUÇÃo

As plantas forrageiras constituem a base da alimentação dos ruminantes, sendo consideradas as fontes que apresentam o mais baixo custo de energia para produtos de origem animal: leite, carne, pele, lã, etc. Entretanto, para o animal, o rendimento energético de tais alimentos constitui um dos principais fatores que limitam o valor nutritivo (Crampton et al., 1960)

O objetivo do presente trabalho é de estudar a digestibilidade "in vitro" da piriquiteira (Trema micrantha Blume) e da gramínea "capim Aripuanã" (Leptochloa domingensis Trin), espécies que crescem espontaneamente na área do Núcleo de Pesquisas de Aripuanä-MT, e do capim-elefante (Pennisetum purpureum Schum.) com vistas a suas potencialidades como recursos forrageiros naquela área.

A composição bromatológica da piriquiteira (Trema micrantha Blume) foi estudada por Andrade et al. (1976). Nesse trabalho, os autores consideraram que essa espécie apresenta uma composição satisfatória para ser utilizada na nutrição animal, contendo teores de fibra

\footnotetext{
( * ) - Trabalho desenvolvido com apoio financeiro do Conselho Nacional de Desenvolvimento Científico e Tecno. lógico (CNPq) e em colaboração com o Instituto Nacional de Pesquisa da Amazônia (INPA) — Projeto Aripuanã.

( *) - Departamento de Melhoramento e Nutrição Animal da Faculdade de Ciências Agrárias e Veterinárias "Campus" de. Jaboticabal - UNESP

(**) - Departamento de Produção Animal da Faculdade de Ciências Agrárias e Veterinárias "Campus" de Jaboticabal - UNESP.

(*.*) - Aluno de Pós-Graduação em Produção Animal e estagiário junto ao Departamento de Melhoramento e Nutrição Animal.
} 
inferiores àqueles de gramíneas tradicionalmente utilizadas como pastagens e teores elevados de proteína bruta.

\section{MATERIAL E MÉTODOS}

\section{OBTENÇÃO DAS AMOSTRAS}

A Trema micrantha Blume, vulgarmente conhecida por periquiteira (em Aripuanã), pau pólvora (em São Paulo e Minas), curumim e chumbinho (ambos na Amazonia), e o capim Aripuanã (Leptochloa domingensis Trin.) foram coletados em Aripuanã em maio de 1976, procedendo em seguida a fenação ao sol. Essas amostras foram devidamente preparadas para as análises bromatológicas.

A amostra do capim elefante (Pennisetum purpureum Schum) foi obtída no laboratório de Nutrição Animal da Faculdade de Ciências Agrárias e Veterinárias de Jaboticabal (SP).

\section{ANÁLISES QUÍMICAS E DIGESTIBILIDADE} "IN VITRO"

As análises químicas foram efetuadas no laboratório de Nutrição Animal da Faculdade de Ciências Agrárias e Veterinárias "Campus" de Jaboticabal - UNESP, para os teores da fibra em detergente neutro (FDN), fibra em detergente ácido (FDA), celulose (Cel.) e lignina (Lig.). A digestibilidade "in vitro" foi conduzida conforme Andrade (1973), com base na técnica de Tilley \& Terry (1963) e Goering \& Van Soest (1970).

Com o objetivo de manter aproximadamente a mesma quantidade de fibra em detergente neutro entre os tratamentos, foram ajustadas as quantidades de amostras para cada substrato.

\section{DELINEAMENTO EXPERIMENTAL}

O ensaio foi conduzido em blocos casualisados, esquema fatorial $4 \times 4$ (4 substratos $x$ 4 tempos) com duplicata por tratamento no bloco.

Para estudar a digestibilidade da fibra, foram determinadas as quantidades de fibra remanescentes após 12, 24, 36 e 48 horas de digestão.

\section{RESULTADOS}

Para a visualização global da composição dos substratos, o Quadro 1 apresenta a composição em \% na matéria seca (M.S.) da foIha de Trema (f.T.), haste + folha de Trema $(h+f-T$.$) , Capim Aripuanã (C.A.) e Capim$ elefante (C.E.).

QUADRO 1 - Composição da folha de Trema, haste + folha de Trema, Capim Aripuanä e Capim elefante \% na Matéria Seca.

FDN FDA Cel. Hem. Lig.

\begin{tabular}{llllll}
\hline Capim Aripuanä & 78,49 & 46,53 & 23,58 & 31,96 & 09,00 \\
folha de Trema & 29,09 & 21,48 & 08,26 & 07,60 & 08,31 \\
$\begin{array}{c}\text { haste+folha de } \\
\text { Trema }\end{array}$ & 38,06 & 36,80 & 20,22 & 01,29 & 13,73 \\
Capim elefante & 60,80 & 36,75 & 28,75 & 16,61 & 04,66 \\
\end{tabular}

As quantidades remanescentes da fibra em detergente neutro (FDA), fibra em detergente ácido (FDA), celulose (Cel.) e Lignina (Lig.) são representadas nos Quadros 2, 3, 4 e 5 respectivamente.

QUADRO 2 - Quantidades remanescentes de fibra em detergente neutro (gramas/tubo), após $12,24,36$ e 48 horas, de tratamento.

\begin{tabular}{ccccc}
\hline & \multicolumn{5}{c}{ TEMPO (horas) } \\
\cline { 2 - 5 } Substratos & $\mathrm{T}_{12}$ & $\mathbf{T}_{24}$ & $\mathbf{T}_{36}$ & $\mathbf{T}_{48}$ \\
\hline & & & & \\
Capim Aripuană & 0,2884 & 0,2536 & 0,2121 & 0,1723 \\
& 0,2929 & 0,2688 & 0,2665 & 0,1744 \\
& & & & \\
folha de Trema & 0,3719 & 0,3689 & 0,4083 & 0,4013 \\
& 0,3409 & 0,3747 & 0,3796 & 0,3345 \\
Trema & & & & \\
haste + folha de & 0,3555 & 0,3429 & 0,3398 & 0,4122 \\
& 0,2722 & 0,3810 & 0,3400 & 0,3226 \\
Capim elefante & 0,3060 & 0,2576 & 0,2793 & 0,1742 \\
& 0,3016 & 0,281 & 0,2016 & 0,1488 \\
& & & & \\
\hline
\end{tabular}


QUADRO 3 - Quantidades remanescentes de fibra em detergente ácido (gramas/tubo) durante 12, 24, 36 e 48 horas de tratamento.

\begin{tabular}{lcccc}
\hline & \multicolumn{5}{c}{ TEMPO (horas) } \\
\cline { 2 - 5 } Substratos & $\mathbf{T}_{12}$ & $\mathbf{T}_{24}$ & $\mathbf{T}_{36}$ & $\mathbf{T}_{48}$ \\
& & & & \\
\hline & & & & \\
Capim Aripuanā & 0,2064 & 0,1749 & 0,1497 & 0,1236 \\
& 0,2032 & 0,1878 & 0,1593 & 0,1280 \\
folha de Trema & 0,3114 & 0,3059 & 0,2842 & 0,2721 \\
& 0,2934 & 0,3020 & 0,3056 & 0,2837 \\
haste + folha de & 0,3954 & 0,3701 & 0,3054 & 0,2884 \\
Trema & 0,4055 & 0,3766 & 0,3670 & 0,2999 \\
& 0,2423 & 0,1717 & 0,1320 & 0,0789 \\
Capim elefante & 0,2198 & 0,1904 & 0,1409 & 0,0912 \\
& & & & \\
\hline
\end{tabular}

QUADRO 4 - Quantidades remanescentes de celulose (gramas/tubo) após 12, 24, 36 e 48 horas de tratamento.

\begin{tabular}{lcccc}
\hline & \multicolumn{5}{c}{ TEMPO (horas) } \\
\cline { 2 - 5 } Substratos & $\mathbf{T}_{12}$ & $\mathbf{T}_{24}$ & $\mathbf{T}_{36}$ & $\mathbf{T}_{48}$ \\
\hline & & & & \\
& & & \\
Capim Aripuanä & 0,1368 & 0,1167 & 0,0972 & 0,0802 \\
& 0,1430 & 0,1293 & 0,1009 & 0,0801 \\
folha de Trema & 0,1393 & 0,1277 & 0,1219 & 0,1319 \\
& 0,1387 & 0,1260 & 0,1335 & 0,1131 \\
haste + folha de & 0,2061 & 0,1904 & 0,1871 & 0,1457 \\
Trema & 0,2520 & 0,2043 & 0,1885 & 0,1642 \\
Capim elefante & 0,1536 & 0,1047 & 0,0815 & 0,0436 \\
& 0,1565 & 0,1305 & 0,0855 & 0,0491 \\
\hline
\end{tabular}

As análises de variâncıa para fibra em detergente neutro, (FDN), fibra em detergente ácido (FDA) e celulose (Cel.). encontram-se no quadro 6 .

Pela análise de variância, ocorrem diferenças entre os substratos; tempo dentro de Capim Aripuanã e elefante e tempo dentro de haste-+ folha de Trema para FDA e celulose. Não se detectaram diferenças significativas entre tempo dentro de folha de Trema e tempo den- tro de haste + folha de Trema, para FDA. Aplicou-se o teste de Tukey para comparação entre médias dos tratamentos. Os Quadros 7 e 8 mostram as comparações entre médias dos substratos e tempo dentro de substratos para fibra em detergente neutro, fibra em detergente ácido e celulose, respectivamente.

QUADRO 5 - Quantidades remanescentes de lignina (gramas/tubo), após $12,24,36$ e 48 horas de tratamento.

\begin{tabular}{lcccc}
\hline & \multicolumn{5}{c}{ TEMPO (horas) } \\
\cline { 2 - 5 } Substratos & $\mathrm{T}_{12}$ & $\mathrm{~T}_{24}$ & $\mathrm{~T}_{36}$ & $\mathrm{~T}_{48}$ \\
& & & & \\
& 0,0548 & 0,0494 & 0,0421 & 0,0353 \\
& 0,0450 & 0,0455 & 0,0453 & 0,0376 \\
Capim Aripuanã & 0,1263 & 0,1345 & 0,1094 & 0,0999 \\
& 0,1117 & 0,1264 & 0,1207 & 0,1272 \\
folha de Trema & & & & \\
& 0,1616 & 0,1550 & 0,1003 & 0,1210 \\
haste + folha de & 0,1211 & 0,1433 & 0,1429 & 0,1136 \\
Trema & 0,0541 & 0,0475 & 0,0330 & 0,0211 \\
Capim elefante & 0,0434 & 0,0408 & 0,0381 & 0,0268 \\
& & & & \\
\hline
\end{tabular}

QUADRO 6 - Análise de variância para as quantidades remanescentes de FDN; FDA e celulose.

\begin{tabular}{|c|c|c|c|}
\hline Causas de Variação & $F(F D N)$ & $F(F D A)$ & F(celulose) \\
\hline Substrato & $59,01^{* * *}$ & $501,54 * * *$ & $168,75 * *$ \\
\hline $\begin{array}{l}\text { Tempo dentro de C. } \\
\text { Aripuanã }\end{array}$ & $7,18^{* *}$ & $17,59 * *$ & $16,26 * *$ \\
\hline $\begin{array}{l}\text { Tempo dentro de folha } \\
\text { de Trena }\end{array}$ & 0,704 NS & $2,00 \mathrm{NS}$ & $1,25 \mathrm{NS}$ \\
\hline $\begin{array}{l}\text { T. dentro de haste }+ \\
\text { folha de Trema }\end{array}$ & $0,422^{\mathrm{NS}}$ & $32,31 * *$ & $22,5 * *$ \\
\hline $\begin{array}{l}\text { Tempo dentro de } \mathrm{C} \text {. } \\
\text { elefante }\end{array}$ & $10,28^{* * *}$ & $59,23 * *$ & 55,00 ** \\
\hline (Tratamentos) & - & - & - \\
\hline Bloco & $1,69 \mathrm{NS}$ & 7,69 & 6,25 \\
\hline Resíduo & - & - & - \\
\hline TOTAL & - & - & - \\
\hline
\end{tabular}

$C V=8,58 \% \quad C V=4,69 \% \quad C V=6,68 \%$ Trema micrantha. . 
QUADRO 7 - Comparação entre as médias dos substratos para fibra em detergente neutro; fibra em detergente ácido e celulose.

\begin{tabular}{ccccc}
\hline Substratos & fr. & $\mathbf{h}+\mathbf{f} . \mathbf{T}$ & $\begin{array}{c}\text { Capim } \\
\text { Aripuanã }\end{array}$ & $\begin{array}{c}\text { Capim } \\
\text { elefante }\end{array}$ \\
\hline FDN & $0,3725^{\circ}$ & $0,3582^{\circ}$ & $0,2373^{\mathrm{b}}$ & $0,2436^{\mathrm{b}}$ \\
$\begin{array}{c}\text { FDA } \\
\text { celulose }\end{array}$ & $0,2947^{\circ}$ & $0,3510^{\mathrm{b}}$ & $0,1666^{\mathrm{c}}$ & $0,1585^{\mathrm{c}}$ \\
\hline
\end{tabular}

QUADRO 8 - Comparaçăo entre as médias para tempos dentro de substratos para fibra em detergente neutro, fibra em detergente ácido e celulose.

\begin{tabular}{|c|c|c|c|c|c|}
\hline Tempc & s (horas) & fT. & $h+f . T$ & $\underset{\text { Aripuanã }}{\text { Capim }}$ & $\begin{array}{c}\text { Capim } \\
\text { elefante }\end{array}$ \\
\hline \multicolumn{6}{|l|}{ FDN } \\
\hline & 12 & $0,3564 a$ & $0,3638^{a}$ & $0,2906^{a}$ & $0,3038 a$ \\
\hline & 24 & $0,3718 a$ & $0,3620^{\circ}$ & $0,2612^{a}$ & $0,2688^{a}$ \\
\hline & 36 & $0,3840^{\circ}$ & $0,3399 a$ & $0,2243^{\circ}$ & $0,2404^{\circ}$ \\
\hline & 48 & $0,3679 a$ & $0,3674^{\circ}$ & $0,1733^{b}$ & $0,1615^{b}$ \\
\hline \multicolumn{6}{|l|}{ FDA } \\
\hline & 12 & $0,2626^{a}$ & $0,4004 a$ & $0,2048 a$ & $0,2310^{a}$ \\
\hline & 24 & $0,3024 a$ & $0,3733^{b}$ & $0,1813^{b}$ & $0,1810^{b}$ \\
\hline & 36 & $0,3040^{a}$ & $0,3362 c$ & $0,1545 c$ & $0,1364 c$ \\
\hline & 48 & $0,2949 a$ & $0,2941 \mathrm{~d}$ & $0,1258 \mathrm{~d}$ & $0,0855 d$ \\
\hline \multirow{4}{*}{ 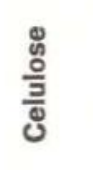 } & 12 & $0,1390^{a}$ & $0,2290 a$ & $0,1399 a$ & $0,1550^{\circ}$ \\
\hline & 24 & $0,1268 a$ & $0,1973 a b$ & $0,1230 a$ & $0,1189 b$ \\
\hline & 36 & $0,1277^{\circ}$ & $0,1878^{c}$ & 0,0990 ab & $0,0835 c$ \\
\hline & 48 & $0,1225^{a}$ & $0,1549 c$ & $0,0801^{b}$ & $0,0463^{d}$ \\
\hline
\end{tabular}

QUADRO 9 - Análise de variância para as quantidades remanescentes de lignina.

\begin{tabular}{cc}
\hline Causas de Variação & F. \\
\hline $\begin{array}{c}\text { Substrato } \\
\text { Tempo } \\
\text { Interaçăo S X T }\end{array}$ & $\begin{array}{c}114,12^{* *} \\
4,12^{*} \\
\end{array}$ \\
\hline (Tratamentos) \\
Bloco \\
Residuo \\
\hline T O T A L & - \\
\hline
\end{tabular}

$\mathrm{CV}=15,59 \%$
Pela análise de variância, há diferença sig. nificativa entre os substratos e entre tempo de fermentação .

Os Quadros 10 e 11 apresentam comparações entre médias de tempo e substratos para lignina.

QUADRO 10 - Comparação entre médias de substratos para lignina.

$$
\text { fT. } \quad h+f . T \quad \underset{\text { Aripuanã }}{\text { Capim }} \begin{array}{r}
\text { Capim } \\
\text { elefante }
\end{array}
$$

Substratos $\quad 0,1195^{a} \quad 0,1323^{a} \quad 0,0444 b \quad 0,0381^{b}$

QUADRO 11 - Comparação entre médias de tempos para lignina.

\begin{tabular}{ccccc}
\hline Tempo (horas) & 12 & 24 & 36 & 48 \\
\hline & $0,0898^{\circ}$ & $0,0928^{\circ}$ & $0,0790^{\circ}$ & $0,0728^{\circ}$ \\
\hline
\end{tabular}

\section{DISCUSSÃo}

Os resultados demonstraram que ocorrem diferenças entre os substratos, quanto a sua degradação "in vitro", sendo que a fibra da Trema, mesmo considerando somente a folha, é muito menos atacada pelos microorganismos que a fibra das gramíneas. Assim, a fibra em detergente neutro apresentou-se praticamente constante ao longo do tempo de fermentação como pode ser observado no Quadro 8 (FDN) . Por outro lado, a fibra em detergente neutro das gramíneas Capim Aripuanã e Capim elefante apresentaram degradação de $40,36 \%$ e $46,84 \%$, respectivamente. Embora a Trema não seja leguminosa, pode comportar-se como tal quanto à digestibilidade da fibra, pois sabe-se que estas apresentam menor degradação que as gramíneas.

Com relação à fração Fibra em detergente ácido, observa-se aproximadamente o mesmo comportamento, porém, para a haste + folha de Trema detectou-se diferenças entre os tempos, 
denotando-se uma degradação. Sabe-se que a diferença entre fibra em detergente neutro (FDN) e fibra em detergente ácido (FDA), daria uma estimativa da hemicelulose. É possível que ocorra alguma substância da FDN da haste + folha de Trema, que não é degradada, o que poderia mascarar a degradação dessa fração, o que não ocorreu com a FDA. Porém a diferença entre os tempos 12 e $48 \mathrm{hs}$. é muito pequena. As gramíneas apresentaram degradação da FDA de 40 a $65 \%$, o que está de acordo com trabalhos "in vitro" de Andrade (1973) em colonião e gordura; e de Rosa \& Andrade (1976) para capim elefante; Schalch \& Andrade (1976) para silagem de milho, "in vitro" e Bertocco et al. (1976), "in vivo".

A fração digestivel da fibra, em detergente ácido, é a celulose, portanto a lignina tem sido considerada não degradável. Fazendo-se um paralelo entre os dados de FDA e celulose, percebe-se que há uma coerência entre a variação dessas frações para a haste + folha de Trema. As gramíneas apresentam degradação para a celulose semelhante àquelas observadas para a fração da FDA.

Os substratos diferem significativamente $(P<0,01)$ quanto aos teores de lignina, o que pode ser observado no Quadro 10. A Trema tem cerca de três vezes mais esse componente que as gramíneas, fato também perfeitamente conhecido, pois sabe-se que as leguminosas forrageiras apresentam essa mesma particularidade e, sendo a Trema uma dicotiledônea arbustiva, pode perfeitamente apresentar um maior teor de lignina, conforme também foi constatado por Andrade et al. (1976).

É provável que a baixa degradação da fibra da Trema seja devido ao alto teor de lignina e também possivelmente à forma de arranjamento da lignina com os outros componentes, especialmente dos carboidratos complexos da fibra da Trema.

Do ponto de vista nutricional podemos considerar que, tendo em vista a baixa degradação microbiana da fibra da Trema, deve-se dar preferência às frações da planta e/ou estágios de maturação (desenvolvimento) que contenham pouca fibra, caso contrário será um alimento de baixa qualidade. Conforme demonstrou Van
Soest \& Wine (1965) a regulação da ingestão de volumoso se dá pelo mécanismo de enchimento quando o alimento apresenta cerca de $60 \%$ ou mais da matéria seca como fibra em detergente neutro. É provável que esse ponto seja o nivel limitante para a utilização da Trema na alimentação dos ruminantes.

\section{ConClusões}

Pelos dados analisados, pode-se concluir que :

1) A Trema apresenta a fibra com baixa degradação pelos microorganismos do rúmem;

2) O capim Aripuanã apresenta uma degradação da fibra semelhante àquela do capim elefante;

3) Essas espécies, que vegetam na área do Núcleo de Pesquisa de Aripuanã. MT, podem ser utilizadas com sucesso na alimentação de bovinos, respeitadas suas características nutricionais.

\section{SUMMARY}

This work with the use of the shrub Trema micrantha Blume and the grass Aripuanā, as possible feed source for livestock. These plants are at A.R.C., MT and were studied through its "in vitro" fiber degradation. Their "in vitro" digestibility was determined in the laboratoties of Faculdade de Ciências Agrárias e Veterinárias de Jaboticabal, São Paulo, the following fractions being analysed: neutral detergent fiber (NDF), acid detergent fiber (ADF), celulose (Cel.) and lignin (Lig.). A sample of elefante grass was used as reference. The results indicated that NDT amount in Trema are maintained during all the fermentation period, whilein the grass the fraction present degra. dation between $40.36 \%$ and $48,84 \%$ in a period of 48 hours. Acid detergent fiber presents the same pattern as that of NDT, the digestibility of this fraction being $40 \%$ and $65 \%$ for Aripuanā grass and elefant grass respectively while in Trema there was no significant difference. A possible explanation would be a higher amount of lignin on Trema, this amount being probably time as that of grasses. Such higher amount of lignin would difficult the microrganisms as lignin presented no degradation during fermentation time. It is concluded that Trema would be useful as feed to ruminant livestock, but its use would depend on a low fiber content because is not digestible. Aripuanā grass apresented the same performance as elefant. 


\section{BIBLIOGRAFIA CITADA}

ANDRADE, P. DE

1973 - Variações dos componentes da parede celular e digestibilidade "in vitro" da fibra das forrageiras Capim Gordura (Melinis minutiflora Pal de Beauv) e Capim Colonião (Panicum maximum Jacq). Tese de Doutoramento, Faculdade de Medicina Veterinária de Jaboticabal.

Andrade, P. de; Vieira, P. de F.; Rosa, L. C. de A.; ANDRADE, T. A.

1976 - Estudos preliminares e composição bromatológica da Trema (Trema micrantha Blume) e do Capim Aripuanã (Leptochloa domingensis Trin). Acta Amazonica, 6(3): Suple. mento : 91-94

Bertocco, J. M.; Vieira, P. de F. \& ANdrade, P. DE

1976 - Constituintes da parece celular e digestibilidade das silagens de três variedades de milho (Zea mays L.). Trabalho apresentado à Faculdade de Medicina Veterinária Agronomia e Zootecnia de Jaboticabal para graduação em Zootecnia.

Crampton, E. W.; Donefer, E. \& Lloyd, L. E

1960 - A nutritive value in dex for forages. J. Anim. Sci., 19: 538-544.

Goering, H. K. \& Van Soest, P. J.

1970 - Forage fiber analyses (Apparantes, reagents, procedures and some applications). Agricultura Research Service.
HARRIS, L. E.

1974 - Compilação de dados analíticos e biológicos para o preparo de tabelas de composiçäo de alimentos para o uso nos trópicos da América Latina. Flórida, Centro de Agricultural Tropical, Universidade of Florida, Gainesville.

Rosa, L. C. \& ANDRADE, P. DE

1976 - Digestibilidade "in vitro" de capim Elefante (Pennisetum purpureaum Shum) em diferentes estadios de maturação. Trabalho apresentado à Faculdade de Medicina Veterinária, Agronomia e Zootecnia de Jaboticabal, para graduação em Zootecnia .

Schalch, E. \& ANDrade, P. DE

1976 - Digestibilidade "in vitro" dos componentes da parede celular e silagens de milho (Zea mays L.). Trabalho apresentado à Faculdade de Medicina Veterinária, Agronomia e Zootecnia de Jaboticabal, para graduação em Zootecnia.

TILley, J. M. A. \& Terry, R. A.

1963 - A two-stage technique for the "in vitro" digestion of forafe crops. J. Br. Grassland Soc., 18: 104-111.

VAN SOEST, P. J. \& Wine, R. H.

1968 - Determination of lignin and celulose in aciddetergent fober with permangnate. Journal A. O.A.C., $51: 780-785$.

(Aceito para publicação em 12/01/78) 